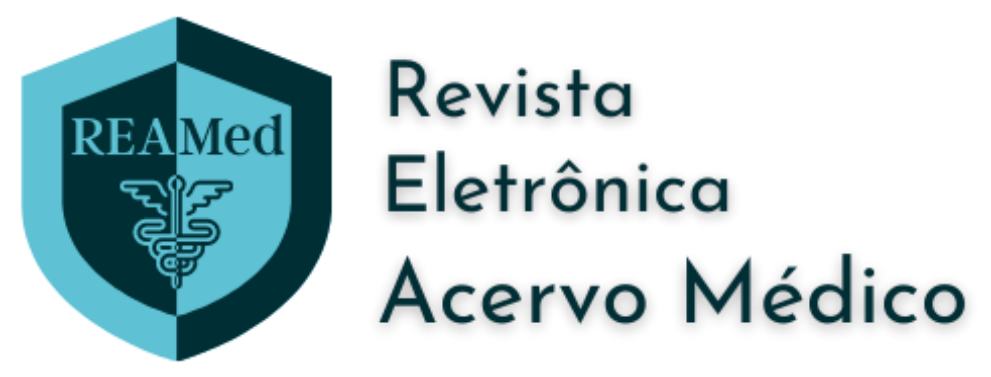

\title{
Efeito do estrogênio no risco cardiovascular: uma revisão integrativa
}

\author{
Estrogen's effect on cardiovascular risk: a integrative review
}

Efecto del estrógeno sobre el riesgo cardiovascular: una revisión integradora

\begin{abstract}
Ellen Larissa Santos da Rocha Maciel ${ }^{1 *}$, Ana Carolina Araújo Mota $^{2}$, Arthur Dias Borges ${ }^{3}$, Giovana Escribano da Costa ${ }^{4}$, John Kennedy Torres de Alencar ${ }^{5}$, Júlia Marques Evangelista ${ }^{6}$, Luiz Valério Costa Vasconcelos ${ }^{5}$, Vitória de Campos Almeida ${ }^{6}$, Adrissa Alana Carneiro Alves ${ }^{6}$, Suzana Tomaz Vasconcelos ${ }^{5}$.
\end{abstract}

\section{RESUMO}

Objetivo: Analisar na literatura científica sobre a relação do estrogênio no risco cardiovascular. Métodos: Essa Revisão Integrativa utilizou plataformas, como PubMed, SciELO, Lilacs e Clinical Trials, para encontrar fontes relevantes para desenvolver este projeto. Descritores em Ciências da Saúde como "cardiovascular risk", "cardiology", "cardiology risk", "estrogen", "estrogen replacement", "menopause", "primary health care" e "hypertension" em associação com operadores booleanos foram usados para pesquisa nessas plataformas científicas, resultando em 2653 artigos. Dentro desses números, devido aos critérios de inclusão e exclusão, apenas 20 tornaram-se fontes oficiais. Resultados: Estudos mostram que o estrogênio tem papel importante na regulação do tônus vascular, evitando a remodelação vascular e, consequentemente, diminuindo o risco cardiovascular. Ainda, a deficiência de estrogênio contribui para o desenvolvimento de hipertensão durante a menopausa devido ao hormônio ovariano vasodilatador circulante reduzido, que causa colapso do sistema circulatório junto com a formação de placas ateroscleróticas. Por outro lado, a reposição hormonal durante a menopausa aumenta a chance de eventos trombóticos. Considerações finais: $O$ estrogênio atua como fator protetor do risco cardiovascular, porém é muito importante considerar outros elementos de risco, como presença de comorbidades, tipos de reposição estrogênica e via de administração de medicamentos, para definir a conduta do paciente.

Palavras-chave Estrogênio, Hipertensão, Menopausa, Risco cardiovascular, Terapia de reposição de estrogênio.

\footnotetext{
1 Universidade Federal do Maranhão (UFMA), Imperatriz - MA. *E-mail: ellenlarissamaciel@gmail.com

${ }^{2}$ Centro Universitário Atenas (UniAtenas), Paracatu - MG.

3 Universidade de Itaúna (UIT), Itaúna - MG.

${ }^{4}$ Universidade Federal do Pará (UFPA), Belém - PA.

${ }^{5}$ Universidade de Fortaleza (Unifor), Fortaleza - CE.

${ }^{6}$ Faculdade Atenas (FA), Sete Lagoas - MG.
}

SUBMETIDO EM: 7/2021

PUBLICADO EM: 8/2021 


\begin{abstract}
Objective: Analyze the scientific literature on the relation of estrogen on cardiovascular risk. Methods: This integrative review used platforms such as PubMed, SciELO, Lilacs and Clinical Trials, to find relevant sources to develop this project. Health Sciences Descriptors like "cardiovascular risk", "cardiology", "cardiology risk", "estrogen", "estrogen replacement", "menopause", "primary health care", and "hypertension" in association with boolean operators were used to search in these scientific platforms, resulting in 2653 articles. Within this number, due to the inclusion and exclusion criteria, only 20 of them became official sources. Results: Studies showed that estrogen has a main role on the regulation of vascular tone, preventing vascular remodeling, therefore, lowering the cardiovascular risk. Also, estrogen deficiency contributes to hypertension during menopause due to the reduced circulating vasodilator ovarian hormone, which causes the circulatory system to collapse along with the formation of atherosclerotic plaques. On the other hand, randomized clinical trials showed us that hormonal replacement during menopause increases the chance of thrombotic events. Final considerations: Estrogen works as a protective factor on cardiovascular risk, however, it's very important to consider other risk elements, like presence of comorbidities, types of estrogen replacement, and drug administration route, to define the patient's approach.
\end{abstract}

Key words: Estrogen, Hypertension, Menopause, Cardiovascular risk, Estrogen replacement therapy.

\title{
RESUMEN
}

Objetivo: Analizar la literatura científica sobre la relación de los estrógenos con el riesgo cardiovascular. Métodos: Esta revisión integradora utilizó plataformas como PubMed, SciELO, Lilacs y Clinical Trials, para encontrar fuentes relevantes para este proyecto. Descriptores de ciências de la salud, como "cardiovascular risk", "cardiology", "cardiology risk", "estrogen", "estrogen replacement", "menopause", "primary health care", y "hypertension" asociados con operadores booleanos se utilizan para buscar en plataformas científicas, resultando en 2653 artículos. De estos, debido a los criterios de inclusión y exclusión, solo 20 son fuentes oficiales. Resultados: Los estudios demostraron que el estrógeno tiene un papel principal, regulando el tono vascular, previniendo la remodelación vascular, luego, reduciendo el riesgo cardiovascular. Además, su deficiencia contribuye a desarrollar hipertensión durante la menopausia debido a la reducción hormonal vasodilatadora ovárica circulante, ocasionando un colapso en el sistema circulatorio y la formación de placas ateroscleróticas. Pero, los ensayos clínicos aleatorios mostraron que el reemplazo hormonal durante la menopausia posibilita eventos trombóticos. Consideraciones finales: El estrógeno actúa como factor protector del riesgo cardiovascular, empero, es importante considerar otros elementos de riesgo, como la presencia de comorbilidades, los tipos de reemplazo hormonal y la vía de administración del fármaco, para centrarse en el paciente.

Palabras clave: Estrógeno, Hipertensión, Menopausia, Riesgo cardiovascular, Terapia de reemplazo de estrógeno.

\section{INTRODUÇÃO}

A Doença Cardiovascular (DCV) é considerada uma das principais causas de morbimortalidade em todo o mundo, sendo a hipertensão uma das formas mais comuns desta patologia (BORGO MV, et al., 2016; SASAKI Y, et al., 2020). Além dos níveis de Pressão Arterial (PA) influenciar no Risco Cardiovascular (RCV), acredita-se que a baixa concentração de estrogênio esteja diretamente relacionada ao aumento das chances de desenvolver doenças cardíacas (CARBONEL AAF, et al., 2020). Relata-se que as mulheres na pósmenopausa, onde há uma queda exponencial de estrogênio, tem maior probabilidade de apresentar quedas pressóricas noturnas em relação a mulheres na pré-menopausa (REAL R e AQUINO N, 2018).

O estrogênio é um hormônio sexual feminino responsável pela liberação, no endotélio, de óxido nítrico e de prostaciclina, substâncias vasodilatadoras. Além disso, ele pode diminuir a produção de endotelina e de angiotensina 2, substâncias vasoconstritoras (NEWSON L, 2018). Ainda, acredita-se que este hormônio 
possa estar, de alguma forma, relacionado ao controle barorreflexo do sistema nervoso simpático e a uma regulação específica dos rins perante a enzima conversora de angiotensina 2 (ECA2). Desse modo, ele seria capaz de proporcionar um controle natural da PA nas mulheres em idade fértil (BOESE AC, et al., 2016). Ademais, a diminuição dos níveis de estrogênios pode influenciar a elevação dos níveis de Lipoproteína de Baixa Densidade (LDL-colesterol) e triglicérides e diminuição da Lipoproteína de Alta Densidade (HDLcolesterol), aumentando o risco para as DCVs (ASSIS IR, et al., 2020).

Antes do período da menopausa, se comparado a homens da mesma idade, as mulheres apresentam pressão arterial mais baixa e, consequentemente, menor risco de desenvolver alterações cardiovasculares (BORGO MV, et al., 2016). No entanto, com a redução dos níveis de estrogênio proporcionados pela menopausa, o risco de doença coronariana aumenta em aproximadamente duas vezes, superando o risco masculino, o que sugere um efeito protetor por esse hormônio, comprovado pelo estudo de Framingham ao comparar a incidência de doença cardiovascular em mulheres antes e depois desse período (NEWSON L, 2018; SASAKI Y, et al., 2020).

Em contrapartida, quando avalia o efeito da Terapia de Reposição Hormonal (TRM) sobre o RCV, percebese, na maioria dos estudos, que prevalece um lado deletério no equilíbrio risco-benefício, principalmente quando a via de administração é a oral. Salienta a necessidade da conduta individualizada para cada paciente, avaliando seu quadro clínico e a janela de intervenção (menor que 10 anos após menopausa). Essa última apresenta uma importância, pois quando inicia a TRM dentro desse intervalo, há uma redução significativa de eventos coronarianos (MOUNIER-VEHIER C, et.al., 2019).

A identificação de novas estratégias para a prevenção e tratamento da hipertensão arterial é de extrema importância no combate das doenças cardíacas e de seus fatores de risco. Diante do exposto, o presente estudo teve como objetivo analisar o que a literatura atual diz a respeito do efeito do estrogênio no risco cardiovascular.

\section{MÉTODOS}

O trabalho consiste em uma Revisão Integrativa e, para a sua elaboração, realizou-se uma busca nas seguintes bases de dados: PubMed, Scientific Electronic Library Online (SciELO), Literatura Latino-americana e do Caribe em Ciências da Saúde (Lilacs) e Clinical Trials. Foram utilizados, de acordo com os Descritores em Ciências da Saúde (DeCS), "cardiovascular risk", "cardiology", "cardiology risk", "estrogen", "estrogen replacement", "menopause", "primary health care" e "hypertension", em associação com os operadores booleanos "AND" e "OR", para fazer a busca dos artigos. A partir dessa pesquisa, foram encontrados 2653 artigos e selecionados 20 artigos completos (Figura 1).

Como filtros, foram usados artigos com resultados compreendidos no período de 2016 a 2021, nos idiomas inglês, português, espanhol e francês e em formato free full text. Excluíram-se os artigos que não corroboram com o tema, não tinham veracidade ou não apresentavam dados relevantes para este estudo. Os critérios de inclusão compreendiam artigos que abordavam a relação entre estrogênio e o risco cardiovascular, incluindo estudos em mulheres no período fértil, na peri e pós menopausa. 
Figura 1 - Resultado da busca bibliográfica.

\begin{tabular}{|l|}
\hline LILACS \\
$\mathrm{N}=111$ \\
\hline
\end{tabular}

\begin{tabular}{|l|}
\hline Pub Med \\
N $=2505$ \\
\hline
\end{tabular}
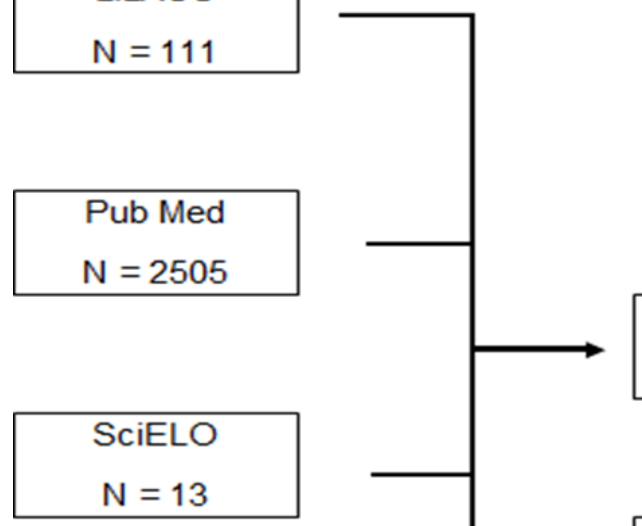

Clinical Trials

$$
N=24
$$

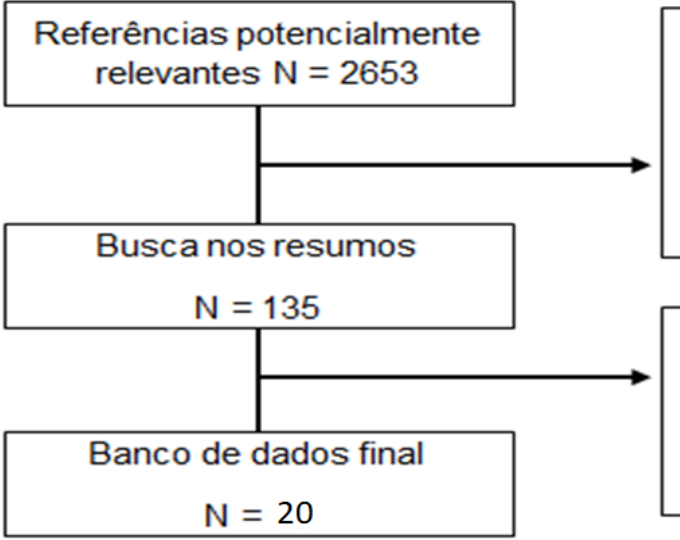

\section{Removidos}

Títulos que não corroboram com o tema

$N=2518$

\section{Removidos}

Não atenderam aos critérios de inclusão

$N=115$

Fonte: Maciel ELSR, et. al., 2021

\section{RESULTADOS}

Após a busca nas bases de dados sobre o tema efeito protetor do estrogênio no risco cardiovascular, foram encontrados 2653 artigos, selecionados os publicados entre 2016 e 2021 e utilizados 20 para compor esta revisão. Os artigos selecionados estão representados no Quadro 1. 
Quadro 1 - Síntese dos principais achados sobre estrogênio e doença cardiovascular dentre os 40 artigos selecionados.

\begin{tabular}{|c|c|c|}
\hline Autores/Ano & Revista & Principais achados \\
\hline $\begin{array}{l}\text { Borgo MV, et al., } \\
2016\end{array}$ & $\begin{array}{l}\text { Braz. J. Med. Biol. } \\
\text { Res. }\end{array}$ & $\begin{array}{l}\text { Estudo experimental analisou histologicamente as artérias coronárias de ratos com hematoxilina e eosina, e foram } \\
\text { analisados parâmetros morfométrico relacionados ao uso de terapia hormonal com estradiol e drospirenona }\end{array}$ \\
\hline $\begin{array}{l}\text { Lovre D et al., } \\
2016\end{array}$ & Sage journals & $\begin{array}{l}\text { Artigo de revisão avaliou que o WHI aumenta os eventos cardiovasculares e câncer de mama em mulheres pós- } \\
\text { menopáusicas. }\end{array}$ \\
\hline $\begin{array}{l}\text { Taylor LE e Sullivan } \\
\text { JC, } 2016\end{array}$ & Journals & $\begin{array}{l}\text { Artigo de revisão examinou os hormônios sexuais que regulam o papel do sistema imunológico no } \\
\text { desenvolvimento da hipertensão e disfunção vascular relacionada a mudanças metabólicas. }\end{array}$ \\
\hline $\begin{array}{l}\text { Boese AC, et } \\
\text { al.,2017 }\end{array}$ & $\begin{array}{l}\text { Am J Physiol Heart } \\
\text { Circ Physiol }\end{array}$ & $\begin{array}{l}\text { Esta revisão descreve diferenças baseadas no sexo na fisiologia e fisiopatologia da vasculatura, com ênfase } \\
\text { especial na sinalização do receptor de esteroides sexuais (receptor de estrogênio e andrógeno) e seu potencial } \\
\text { impacto na função vascular na saúde e doenças (por exemplo, aterosclerose, hipertensão, doença arterial, } \\
\text { aneurismas de aotorismo abdominal, aneurismas cerebrais e derrame). }\end{array}$ \\
\hline $\begin{array}{l}\text { Manosroi W, et al., } \\
2017\end{array}$ & $\begin{array}{l}\text { J Clin Endocrinol } \\
\text { Metab }\end{array}$ & $\begin{array}{l}\text { As análises multivariadas de genes e dados do HyperPATH entre variantes de ESR2 e SSBP em mulheres e } \\
\text { homens normotensos e hipertensos }\end{array}$ \\
\hline $\begin{array}{l}\text { Manson JE, et al., } \\
2018\end{array}$ & JAMA & $\begin{array}{l}\text { Este ensaio clínico busca avaliar o efeito da terapia com CEE associada à MPA sobre o risco de mortalidade } \\
\text { cardiovascular e por câncer. }\end{array}$ \\
\hline Melo JB, et al., 2018 & Int. J. Cardiovasc. & $\begin{array}{l}\text { Este ensaio clínico randomizado busca avaliar a relação entre a prevenção de calcificação de artéria coronária e } \\
\text { o menor risco de morbidade e mortalidade de mulheres pós - menopausa. }\end{array}$ \\
\hline Mir D, et al., 2018 & Cureus & $\begin{array}{l}\text { Estudo de caso que concluiu que o uso crônico de contraceptivos orais aumentará ligeiramente a pressão } \\
\text { sistêmica na maioria das mulheres. É aconselhável evitar o ACO em pacientes de alto risco e fazer verificações } \\
\text { regulares de PA em pacientes com ACO. }\end{array}$ \\
\hline $\begin{array}{l}\text { Newson L, et al., } \\
2018\end{array}$ & Post Reprod Health & $\begin{array}{l}\text { Esta revisão de literatura busca apresentar um panorama geral dos fatores de risco mais importantes para } \\
\text { doenças cardiovasculares e como gerenciar esses fatores de risco de forma adequada, com base nas evidências } \\
\text { disponíveis. }\end{array}$ \\
\hline $\begin{array}{l}\text { Real R e Aquino MN, } \\
\qquad 2018\end{array}$ & An. Fac. Cienc. Méd & $\begin{array}{l}\text { Esta revisão de literatura buscou descrever os mecanismos fisiopatológicos da hipertensão arterial na menopausa } \\
\text { e os medicamentos anti-hipertensivos atualmente recomendados. }\end{array}$ \\
\hline Swica Y, et al., 2018 & Menopause & $\begin{array}{l}\text { O estudo relatou que o CEE administrado VO com ou sem MPA, predispõe um risco aumentado de hipertensão } \\
\text { em mulheres pós-menopáusica mais velha. }\end{array}$ \\
\hline $\begin{array}{l}\text { ACUNA M, et al., } \\
2019\end{array}$ & $\begin{array}{l}\text { Revista Chilena de } \\
\text { Ginecologia e } \\
\text { Obstetrícia }\end{array}$ & $\begin{array}{l}\text { Revisão de literatura que concluiu que a TRM melhora a qualidade de vida das mulheres no climatério e na } \\
\text { menopausa. O estrogênio tem melhores benefícios nas doenças cardiovasculares, administrando a terapia } \\
\text { precoce após a menopausa e mantendo-a por um longo tempo. }\end{array}$ \\
\hline
\end{tabular}

REAMed | Vol. 1(1) | DOI: https://doi.org/10.25248/REAMed.e8527.2021

Página 5 de 10 


\begin{tabular}{|c|c|c|}
\hline Autores/Ano & Revista & Principais achados \\
\hline $\begin{array}{l}\text { Mounier-Vehier C, et } \\
\quad \text { al., } 2019\end{array}$ & La Presse Médicale & $\begin{array}{l}\text { Revisão de literatura que avaliou que se uma TRH for prescrita a uma mulher hipertensa controlada com risco } \\
\text { cardiovascular baixo a moderado, o ginecologista e o médico cardiovascular devem reavaliar regularmente o risco } \\
\text { CV e garantir o controle dos fatores de risco modificáveis (hipertensão, hipercolesterolemia LDL, açúcar no } \\
\text { sangue, tabagismo). Eles devem encorajar seu paciente a respeitar o melhor possível uma ótima higiene de vida. }\end{array}$ \\
\hline Zhu Q, et al., 2019 & BMC & Estudo entre a relação do E2 e os fatores de risco de DCV entre as mulheres pós-menopausa centenárias. \\
\hline Assis IR, et al., 2020 & $\begin{array}{l}\text { Rev Med Minas } \\
\quad \text { Gerai }\end{array}$ & $\begin{array}{l}\text { Estudo prospectivo observacional do tipo caso controle realizado com } 224 \text { mulheres hipertensas atendidas na } \\
\text { cidade de Barbacena-MG avaliou a influência do climatério sobre o controle da pressão arterial em mulheres } \\
\text { hipertensas. }\end{array}$ \\
\hline $\begin{array}{l}\text { Carbonel AAF, et al., } \\
\qquad 2020\end{array}$ & $\begin{array}{l}\text { Rev. Assoc. Med. } \\
\text { Bras }\end{array}$ & $\begin{array}{l}\text { Esta revisão aborda a relação entre a terapia hormonal com estradiol e a modulação da função vascular, o que } \\
\text { estimula a liberação de compostos vasoativos, que deslocam o eixo da angiotensina para produção de } \\
\text { angiotensinogênio. }\end{array}$ \\
\hline $\begin{array}{l}\text { Sabbatini AR e } \\
\text { Kararigas GK, } 2020\end{array}$ & Biol Sex Differ & $\begin{array}{l}\text { Este artigo, discute o papel do E2 nos mecanismos que contabilizam o desenvolvimento de hipertensão arterial e } \\
\text { de danos de órgãos alvo de forma específica de acordo com o sexo. }\end{array}$ \\
\hline Sasaki Y, et al., 2020 & $\begin{array}{l}J \text { Atheroscler } \\
\text { Thromb }\end{array}$ & $\begin{array}{l}\text { Este estudo investigou se a expressão de SIRT1 está envolvida na etiologia da senescência arterial induzida pela } \\
\text { menopausa e no desenvolvimento aterosclerótico. }\end{array}$ \\
\hline Mehta J, et al., 2021 & $\begin{array}{l}\text { Frontiers em } \\
\text { endocrinologia }\end{array}$ & $\begin{array}{l}\text { Este ensaio clínico analisa a relação entre riscos da terapia hormonal e idade da mulher, bem como o tempo de } \\
\text { início da menopausa. }\end{array}$ \\
\hline $\begin{array}{l}\text { Noah MLN, et al., } \\
2021\end{array}$ & Frontiers & $\begin{array}{l}\text { O artigo relatou que a terapia de reposição E2 é usada para controlar os sintomas da menopausa e minimizar a } \\
\text { incidência de doenças cardiovasculares em mulheres na pós-menopausa. }\end{array}$ \\
\hline
\end{tabular}

Fonte: Maciel ELSR, et. al., 2021 


\section{DISCUSSÃO}

\section{A ação do estrogênio no sistema cardiovascular}

Sabe-se que o estrogênio pode modular a função vascular ao se ligar aos receptores das células endoteliais e do músculo liso vascular, induzindo a liberação de óxido nítrico e prostaciclinas, ambos vasodilatadores (CARBONEL AAF, et al., 2020).

Estudos clínicos e experimentais têm demonstrado que o estrogênio regula o tônus vascular induzindo a vasodilatação, impede processos de remodelação vascular impossibilitando o crescimento de células musculares lisas vasculares, cessa a resposta vascular à lesão, bloqueia o sistema renina-angiotensinaaldosterona, inibe a endotelina, fornece proteção renal, diminui o tônus simpático basal e estimula a biodisponibilidade do óxido nítrico. Em contrapartida, a deficiência de estrogênio contribui para a hipertensão arterial na menopausa devido a um colapso do sistema circulatório, deficiência de hormônios ovarianos vasodilatadores coincidindo com 0 surgimento de fatores aterogênicos, síndrome metabólica e hipersensibilidade ao sal (MELO JB, et al., 2018; SABBATINI AR e KARARIGAS G, 2020).

Sob diferente análise, o estrogênio desempenha um papel protetor no desenvolvimento da hipertensão e de lesões de órgão-alvo, exercendo diversos efeitos modulatórios. A diminuição do estrógeno leva a alterações nos mecanismos de regulação do sistema nervoso simpático, sistema renina-angiotensinaaldosterona, massa corporal, estresse oxidativo, função endotelial e sensibilidade ao sal; todos associados a um estado inflamatório crucial e influenciados por fatores genéticos, resultando em danos cardíacos, vasculares e renais na hipertensão (SABBATINI AR e KARARIGAS G, 2020).

Os efeitos do estrogênio no sistema cardiovascular são mediados por dois subtipos de receptor de estrogênio (ER), ER- $\alpha$ (codificado pelo gene ESR1) e ER- $\beta$ (codificado pelo gene ESR2). Polimorfismos no ER têm sido associados ao risco de doenças cardiovasculares em homens e mulheres. ESR2 é altamente expresso na vasculatura, e a ligação do estrogênio a este receptor geralmente leva à vasodilatação. Sua ativação pode proteger contra o efeito adverso da doença cardiovascular. Além disso, relata que mulheres heterozigotas para certos polimorfismos genotípicos de ESR2 apresentam risco aumentado de hipertensão, especialmente as que usam anticoncepcionais orais, sugerindo que esses polimorfismos podem transformar a interação do estrogênio com ESR2 de um efeito protetor sobre a pressão arterial para um prejudicial (MANOSROI W, et al., et al., 2017).

O estrogênio tende a reduzir a ingestão de gordura e aumentar o gasto de energia para limitar o acúmulo de gordura corporal e, portanto, representa um elo importante para a prevenção da morbidade cardiovascular induzida pela obesidade. Além disso, atuam como um fator determinante na inflamação crônica, conforme demonstrado pela prevalência de doenças autoimunes entre as mulheres e a variabilidade do estado inflamatório durante o ciclo menstrual, gravidez e menopausa. A atividade imunossupressora do estrogênio tem sido bem estabelecida em vários modelos de doença inflamatória e, em geral, o 17- $\beta$ estradiol inibe a diferenciação de células de macrófagos pró-inflamatórios Th17 e M1 e aumenta a expansão de macrófagos Treg e M2. Assim, a sinalização do estradiol 17- $\beta$ pode desempenhar um papel fundamental na promoção de células imunes anti-inflamatórias para proteção contra hipertensão e disfunção vascular (TAYLOR LE e SULLIVAN JC, 2016).

Pelos efeitos adaptativos do estrogênio, as mulheres têm menor risco de desenvolver doenças cardiovasculares e suas complicações associadas à sua idade reprodutiva. $\mathrm{O}$ menor risco dessas patologias em mulheres na pré-menopausa poderia ser atribuído ao estrogênio mantendo a pressão arterial através da regulação indireta do perfil lipídico e aumentando as atividades de óxido nítrico. Dessa forma, há uma prevenção de insultos cardíacos durante o estresse por vias de sinalização direta e indireta, conferindo vaso proteção e regulação oportuna da resposta pró e anti-inflamatória (NOAH MLN, et al., 2021).

\section{Os efeitos da menopausa no sistema cardiovascular}

Observa-se que a chegada do climatério nas mulheres associa-se a um aparecimento de quadro hipertensivo e a vários outros riscos cardiovasculares. É nesta fase que acontece a redução da produção do estrógeno e aumento dos níveis de andrógenos. Portanto, reafirma-se que as mulheres no climatério 
apresentam uma diminuição da produção de estrógenos e um aumento do nível de andrógenos circulantes. Esses dois hormônios atuam no sistema nervoso simpático, na função cardiovascular, no equilíbrio hidroeletrolítico e no sistema renina angiotensina, acarretando em alterações nos níveis pressóricos. Assim, a diminuição dos níveis de estrogênios pode influenciar na elevação dos níveis de LDL e triglicérides e na diminuição de HDL, aumentando o risco para as doenças cardiovasculares (ASSIS I, et al., 2020).

O estradiol (E2) pode regular a taxa de síntese da apolipoproteína estrutural HDL no fígado. Ele aumenta o HDL, aumentando a taxa de síntese de apolipoproteína A-I (apoA-I) e apolipoproteína A-II (apoA-II). Há uma grande discrepância no nível de HDL em mulheres na pós-menopausa. Alguns estudos mostraram uma diminuição significativa nos níveis de HDL em mulheres na pós-menopausa, enquanto outros estudos mostraram um aumento significativo nos níveis de HDL. O resultado dessa diferença pode ser devido à variação na população do estudo, intervenções na vida e duração da menopausa (ZHU Q, et al., 2019).

Por outro lado, HDL possui diferentes tipos de subtipos. Estudos mostraram que a gravidade da doença cardiovascular estava relacionada à distribuição dos subtipos de HDL. Grandes partículas de HDL2b diminuíram significativamente, enquanto pequenas partículas de pré-HDL e HDL3 aumentaram. O nível do subtipo HDL3 em mulheres pós-menopáusicas é maior do que antes da menopausa, sugerindo que os hormônios sexuais podem afetar a distribuição dos subtipos $\mathrm{HDL}$, o que pode estar relacionado ao rápido aumento do risco de doença cardiovascular em mulheres após a menopausa (CARBONEL AAF, et al., 2020; ZHU Q, et al., 2019).

Nota-se que a prevalência da hipertensão arterial aumenta de acordo com pessoas acima de 60 anos. As mulheres na menopausa giram em torno de $25 \%$ dessa população e, antes da menopausa, a porcentagem é menor sendo independente de sua raça. Percebe-se que a patogênese da hipertensão é complexa; o estrogênio regula o tônus vascular, assim induz a vasodilatação, previne a remodelação vascular quando inibe o crescimento das células do músculo liso vascular, ocorre também a inibição da resposta vascular à lesão e o sistema renina-angiotensina. Enquanto a aldosterona inibe a endotelina, fornecendo a redução do tônus simpático basal e estimula a biodisponibilidade do óxido nítrico que favorece a vasodilatação (REAL $R$ e AQUINO N, 2018).

Conforme o resultado do estudo realizado em Barbacena no ano de 2018, percebe-se que as mulheres na pós-menopausa apresentaram maior risco de ocorrência em eventos cardiovasculares em até 10 anos, o qual foi indicado pelo cálculo do escore de Framingham (utilizado para estratificar o risco cardiovascular de acordo com os fatores de risco: sexo, idade, dosagem do LDL e HDL, pressão arterial, tabagista, diabético); mas o risco aumentando não estava diretamente ligado aos níveis séricos de HDL, triglicerídeos ou a medida da circunferência abdominal. Desse modo, reforçou a ideia da transição menopáusica, que é marcada pelo período de várias alterações metabólicas complexas (ASSIS I, et al., 2020).

\section{Os efeitos cardiovasculares da terapia hormonal com estrogênio}

A Terapia de Reposição Hormonal é o tratamento mais eficaz para o alívio dos sintomas vasomotores, como ondas de calor e sudorese noturna nas mulheres no climatério e na menopausa (MEHTA J, et al., 2021). E para milhões de mulheres, as pílulas anticoncepcionais orais são uma maneira eficaz de prevenir uma gravidez indesejada, regular período menstrual e tratamento de problemas devido a mudanças hormonais, como acne (MIR D, et al., 2018).

Sendo assim os tratamentos com estrogênios, como a terapia de reposição hormonal, os anticoncepcionais e terapias tópicas, têm sido objeto de muita discussão e pesquisa desde os anos sessenta. Com o envelhecimento da população, a disseminação do conhecimento sobre métodos contraceptivos e do conhecimento de riscos à saúde da mulher com uso desses hormônios, inúmeros estudos começaram a ser desenvolvidos e repetidos no decorrer das décadas, permitindo a evolução destes tratamentos orientada pela mudança na compreensão dos riscos e benefícios (LOVRE D, et al., 2017; MEHTA J, et al., 2021).

Os primeiros resultados de grandes ensaios randomizados sobre terapia de reposição hormonal na menopausa alertaram para o aumento de eventos cardiovasculares em caso de prescrição por via oral (MOUNIER-VEHIER C, et.al., 2019). Análises adicionais mostraram a importância da janela de intervenção, 
ou seja, em até 10 anos após início da menopausa- para iniciar a terapia hormonal, considerando as comorbidades pré-existentes e o tipo de terapia realizada, podendo ser dos tipos orais, transdérmicos, tópicos, vaginal, combinados com progesterona e dentre outros (MIR D, et al., 2018; SWICA Y, et al., 2018; MOUNIERVEHIER C, et al., 2019; MEHTA J, et al., 2021).

O estrogênio oral, usado tanto em pílulas anticoncepcionais e para terapia de reposição hormonal na menopausa, possui riscos decorrentes, em grande parte, do metabolismo de primeira passagem pelo fígado, pois aumenta a produção de fatores de coagulação e marcadores inflamatórios. Além disso, pode causar hipertrigliceridemia, aumento do risco de tromboembolismo venoso e cálculos biliares, diferente dos transdérmicos e dos tópicos vaginais (MEHTA J, et al., 2021).

Os estrogênios têm grande benefício na doença cardiovascular e na redução da mortalidade total, se iniciados na janela de oportunidade, tendo evidência menos forte ao combinar estrogênios com progestágenos (MANSON JE, et al., 2018; ACUNÃ M, et al., 2019).

Estudos demonstram que a terapia hormonal reduz a incidência de diabetes mellitus, auxilia na prevenção e redução da placa de ateroma, na redução do risco de infarto agudo do miocárdio e melhora função pósisquemia, devido a ação vasodilatador e antioxidante do estrogênio, porém, não é indicado para cardioproteção secundária (ACUNÃ M, et al., 2019). Também é encontrado na literatura, propriedades benéficas na homeostase metabólica, incluindo equilíbrio energético devido à diminuição do LDL e aumento do HDL (LOVRE D, et al., 2017).

Em relação a associação da terapia com estrogênio e o desenvolvimento da hipertensão arterial, os estudos são controversos, tendo a sua maioria relacionado o estrogênio por via oral, com ou sem progestágeno, a um aumento do risco de hipertensão em mulheres mais velhas na pós-menopausa. Esse mecanismo também ocorre devido ao metabolismo hepático de primeira passagem, que leva ao aumento da produção de Proteína C-reativa (PCR) e angiotensina II e à redução do IGF-I, processos implicados na patogênese da hipertensão (MIR D, et al., 2018; SWICA Y, et al., 2018).

Aumentos na pressão arterial, no entanto, não ocorrem em todas as mulheres que tomam estrogênio. Alguns estudos sugerem que os efeitos do estrogênio na pressão arterial podem ser geneticamente mediados (SWICA Y, et al., 2018). Sendo assim, é aconselhável evitar o uso da terapia oral em pacientes de alto risco e fazer verificações regulares da pressão arterial e acompanhamento médico (MIR D, et al., 2018).

\section{CONSIDERAÇÕES FINAIS}

Portanto, é consenso entre a literatura comparada que o estrogênio atua como fator protetor do risco cardiovascular, devido a ações como a estimulação da liberação de substâncias vasodilatadoras e a inibição da vasoconstrição do endotélio. No entanto, é importante ressaltar que a eficácia do estrogênio está muito relacionada ao hormônio fisiológico e que a eficácia da terapia hormonal pós - menopausa, no sentido de prevenir ou tratar doenças como hipertensão e aterosclerose, depende das condições de uso. Assim, é importante considerar outros elementos de risco, como presença de comorbidades, tipos de reposição estrogênica e via de administração de medicamentos, para definir a conduta do paciente. Nosso trabalho visa sintetizar sobre o efeito do estrogênio do RCV, coletando dados mais atuais da literatura, para facilitar no estudo do tema. No entanto, ressaltamos que mais estudos são necessários para compreender melhor o assunto.

\section{REFERÊNCIAS}

1. ASSIS IR, et al. The effects of climateric on systemic blood pressure. Revista Médica de Minas Gerais, 2020; 30: 525532.

2. ACUÑA M, et al. Estrogen therapy and primary cardiovascular prevention. Revista Chilena de Obstetrícia e Ginecologia, 2019; 84: 514-524.

3. BOESE AC, et al. Sex differences in vascular physiology and pathophysiology: estrogen and androgen signaling in health and disease. Am J Physiol Heart Circ Physiol, 2017; 1: 524-545. 
4. BORGO MV, et al. Hormonal therapy with estradiol and drospirenone improves endothelium-dependent vasodilation in the coronary bed of ovariectomized spontaneously hypertensive rats. Brazilian Journal of Medical and Biological Research, 2015; 49.

5. CARBONEL AAF, et al. Cardiovascular system and estrogen in menopause. Revista da Associação Médica Brasileira, 2020; 66: 97-98.

6. LOVRE D, et al. Effect of menopausal hormone therapy on components of the metabolic syndrome. Ther Adv Cardiovasc Dis, $2016 ; 11: 33-43$.

7. MANOSROI W, et al. The association of estrogen receptor- $\beta$ gene variation with salt-sensitive blood pressure. J Clin Endocrinol Metab, 2017; 1: 4124-4135.

8. MANSON JE, et al. Terapia hormonal menopáusica e mortalidade por todas as causas e causas específicas a longo prazo: Ensaios randomizados da Women's Health Initiative. JAMA, 2017; 318: 927-938.

9. MELO JB, et al. Fatores de risco cardiovasculares em mulheres climatéricas com doença arterial coronariana. Int $\mathrm{J}$ Cardiovasc Sc, 2018; 31:4-11.

10. MEHTA J, et al. Risks, benefits, and tratment modalities of menopausal hormone therapy: current concepts. Frontiers in Endocrinology, 2021;12.

11. MIR D, et al. Malignant hypertension in association with low estrogen dose oral contraceptives: Case report and review of literature. Cureus, 2018; 13: e2978

12. MOUNIER-VEHIER C, et al. HTA et traitement hormonal de la menopause. La Presse Médicale, 2019: 1295-1300

13. NOAH MLN, et al. Sex-gender disparities in cardiovascular diseases: The effects of estrogen on eNOS, lipid profile, and NFATs during catecholamine stress. Frontiers in Cardiovascular Medicine; 2021: 8.

14. NEWSON L. Menopausa e doenças cardiovasculares. Post Reproductive Health, 2018; 24: 44-49.

15. REAL R, M AQUINO M. Treatment of arterial hypertension in menopause. Anales de La Facultad de Ciencias Médicas (Asunción), 2018; 51: 65-74.

16. SABBATINI AR, KARARIGAS G. Estrogen-related mechanisms in sex differences of hypertension and target organ damage. Biology of Sex Differences, 2020; 11.

17. 1SASAKI Y, et al. Estrogen-SIRT1 axis plays a pivotal role in protecting arteries against menopause-induced senescence and atherosclerosis. J Atheroscler Thromb, 2020; 27 :47-59.

18. SWICA Y, et al. Effects of oral conjugated equine estrogens with or without medroxyprogesterone acetate on incident hypertension in the Women's Health Initiative hormone therapy trials. Menopause, $2018 ; 25: 753-761$.

19. TAYLOR LE, SULLIVAN JC. Sex differences in obesity-induced hypertension and vascular dysfunction: a protective role for estrogen in adipose tissue inflammation? Am J Physiol Regul Integr Comp Physiol, 2016 ;311:14-20.

20. ZHU Q, et al. Oestradiol: any role in cardiovascular risk factors in female centenarians of Hainan? BMC Cardiovasc Disord, 2019; $16: 115$. 